
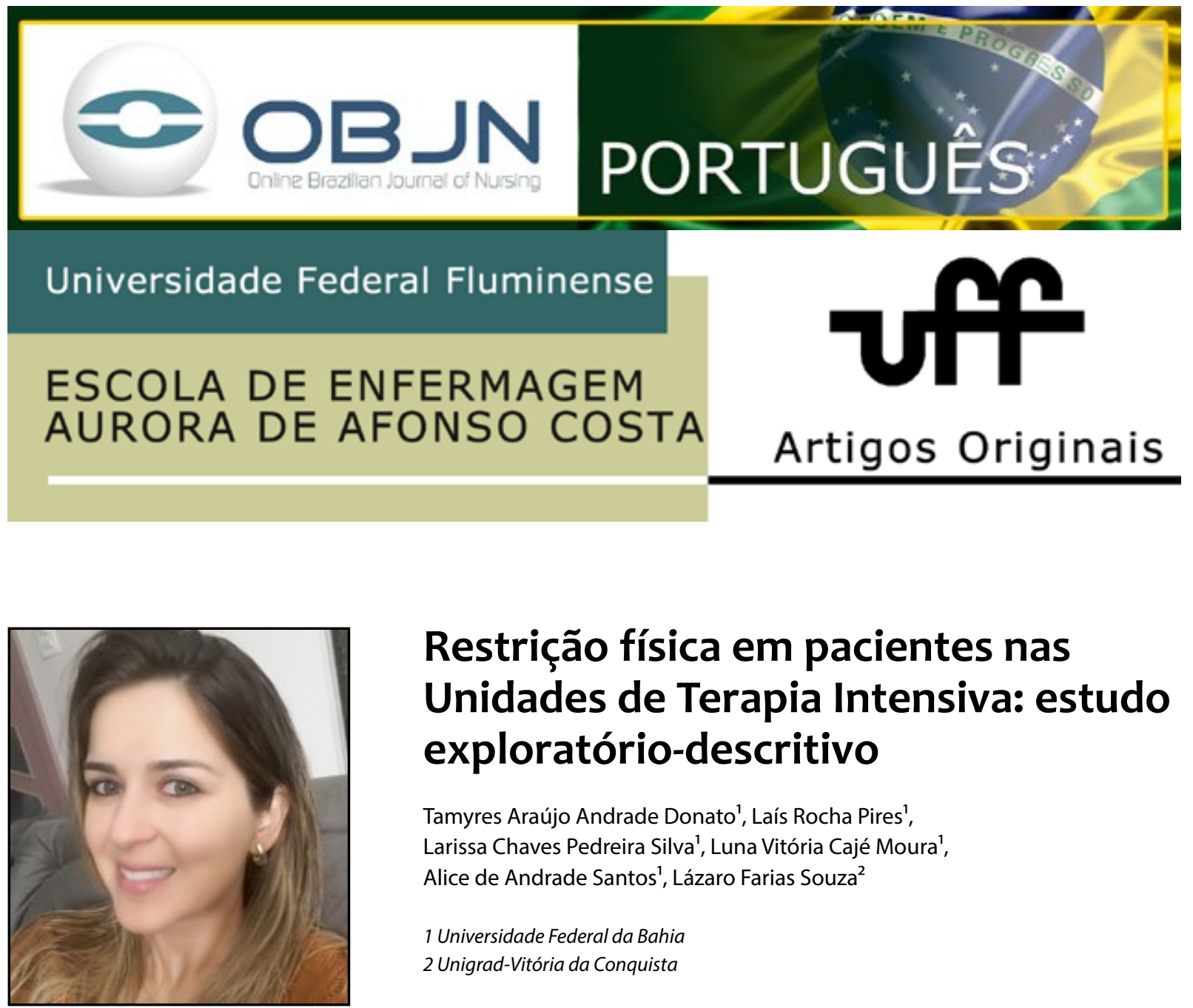

\title{
Restrição física em pacientes nas Unidades de Terapia Intensiva: estudo exploratório-descritivo
}

\author{
Tamyres Araújo Andrade Donato', Laís Rocha Pires', \\ Larissa Chaves Pedreira Silva', Luna Vitória Cajé Moura', \\ Alice de Andrade Santos ${ }^{1}$, Lázaro Farias Souza ${ }^{2}$ \\ 1 Universidade Federal da Bahia \\ 2 Unigrad-Vitória da Conquista
}

\section{RESUMO}

Objetivo: analisar os critérios para uso e monitorização de restrições físicas em pacientes internados na Unidade de Terapia Intensiva (UTI). Método: a pesquisa teve caráter exploratório, descritivo e qualitativo e foi realizada em duas UTIs da Bahia, com 85 profissionais de enfermagem. Os dados foram organizados com base na análise temática. Resultado: a equipe de enfermagem justifica a utilização da restrição física para a segurança do paciente. Como critério para seu uso, verificam-se o nível de consciência, o grau de agitação e/ou a desorientação. Para a monitorização, observam-se a integridade cutânea e as alterações do nível de consciência. Por isso, a avaliação neurológica foi a técnica mais empregada pelos profissionais para verificar a necessidade de restrição. $O$ conhecimento da equipe em relação aos instrumentos legais que regem esse procedimento se mostrou superficial. Conclusão: identificaram-se fragilidades nos critérios de monitorização e suspensão da restrição física, visto que o conhecimento ainda é incipiente e que não há protocolos definidos.

Descritores: Restrição Física; Pacientes; Enfermagem; Unidade de Terapia Intensiva. 


\section{INTRODUÇÃO}

Paciente crítico ou grave é aquele que se encontra em risco iminente de perder a vida ou a função de algum órgão/sistema do corpo humano, ou seja, aquele que se encontra em frágil condição clínica, que requer cuidado

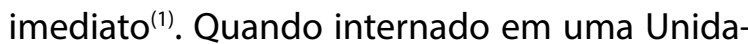
de de Terapia Intensiva (UTI), ele se utiliza de monitorização multiparamétrica contínua, além de diversos artefatos terapêuticos para manejo eficaz de seu quadro. Tais pacientes podem, de forma intencional ou não, remover dispositivos como tubos endotraqueais, acessos vasculares, sondas. Além disso, existe um risco de ocorrências de quedas quando confusos, desorientados ou agitados. Diante disso, muitas vezes, torna-se necessário utilizar alguma forma de proteção para garantir sua segurança.

Um dos métodos mais comuns para garantir segurança ao paciente crítico é o uso de restrições físicas ${ }^{(2)}$, identificadas também como contenções mecânicas ou contenções físicas, que se configuram como um método restritivo da liberdade de movimento da pessoa, da mobilidade física ou do acesso normal ao seu corpo ${ }^{(3)}$.

As restrições mais utilizadas são aquelas instaladas nos membros superiores, exceto em terapia intensiva, na qual as restrições de pulso são as mais utilizadas por meio de curativos e faixas de gaze ${ }^{(2)}$.

No que se refere ao seu uso, esse método deve ser empregado em casos de necessidade de proteção ao paciente contra lesões, traumas, quedas, deslocamento de dispositivos e outros. Ademais, serve para prevenir a interrupção do tratamento a que o enfermo vem sendo submetido ${ }^{(4)}$. Sua utilização, entretanto, também tem sido frequente em situações de agitação, embora se recomende, primeiramente, a identificação da causa, a fim de se avaliarem alternativas de tratamento antes da restrição(5).
O emprego de restrições físicas é prática comum no Brasil, porém controversa, tendo em vista o uso frequente e desenfreado dessa técnica sem avaliação, de caráter coercitivo/punitivo, bem como suas consequências. Tal método, em diversos países como Itália, Índia, Finlândia, Alemanha, Suíça e Estados Unidos, apresenta uma taxa de prevalência que varia entre 0,25\% e 59\%, envolvendo pacientes psiquiátricos no setor de emergência. Constatam-se a menor prevalência de seu uso na Itália, de 0,25\%, e a maior, de 59\%, nos Estados Unidos ${ }^{(6)}$. Em países como Reino Unido e Holanda, seu uso é proibido ${ }^{(5)}$.

No Brasil, a resolução 427/2012 do ConseIho Federal de Enfermagem (COFEn) proíbe sua execução com propósito de disciplina, punição e coerção ou por conveniência da instituição ou da equipe de saúde ${ }^{(7)}$. Ressalta-se, ainda, que as instituições, preferencialmente, devem estabelecer protocolos assistenciais com o intuito de regular o uso da restrição física e o monitoramento do paciente contido, ficando a utilização desse dispositivo de segurança atrelada ao objetivo único de prevenir dano imediato ou iminente ao paciente ou aos demais ${ }^{(7)}$.

Durante a prática assistencial em terapia intensiva, observou-se a grande quantidade de restrições físicas aplicadas sem discussão quanto aos critérios para seu uso, sua retirada ou seu monitoramento, a despeito das complicações que essa técnica possa causar. No Brasil, discussões sobre a segurança do paciente vêm ocorrendo, culminando no Programa Nacional de Segurança do Paciente, em $1^{\circ}$ de abril de $2013^{(8)}$, com a implantação da gestão de risco e de Núcleos de Segurança do Paciente nos estabelecimentos de saúde; o que levantou a inquietação sobre os critérios de uso e de monitorização dessas restrições na UTI, em que o paciente já se encontra fragilizado por sua doença e pelo ambiente que o cerca. 
A partir de buscas referentes às produções dos últimos cinco anos em bases de dados científicas nacionais e internacionais, percebe-se que a produção no âmbito da enfermagem, de um modo geral, costuma dar maior enfoque para as técnicas de restrição e seus cuidados, contudo questões relativas aos critérios para o uso e para a sua monitorização têm sido brevemente discutidas, tendo um foco maior na área de psiquiatria.

Em se tratando do paciente crítico, pouco tem sido abordado, mesmo sendo esse procedimento de uso frequente nas UTIs. Diante desse contexto, questiona-se: Quais os critérios da equipe de enfermagem para o uso das restrições físicas no paciente crítico? Como é realizada a sua monitorização?

O objetivo do estudo foi analisar os critérios para uso e monitorização de restrições físicas em pacientes internados na UTI.

Pretende-se, com essa análise, dar visibilidade ao tema, mostrando como ele vem sendo tratado pelos profissionais da enfermagem na terapia intensiva, a fim de proporcionar discussões que possam dar mais segurança ao uso das restrições físicas, evitando restrições prolongadas, desnecessárias e eventos adversos relacionados a elas.

\section{MÉTODO}

Pesquisa exploratório-descritiva, com abordagem qualitativa, realizada em duas UTIs do estado da Bahia: uma situada em Salvador e outra em Vitória da Conquista, denominadas, a partir de então, como UTI A e UTI B, respectivamente. $O$ critério de escolha das unidades deu-se a partir do perfil de pacientes atendidos, em sua maioria críticos de alta complexidade.

A UTI A situa-se em um hospital privado de médio porte no município de Salvador, possui
30 leitos: seis destinados a pacientes críticos de baixa complexidade e 24 para pacientes de alta complexidade terapêutica. O perfil da unidade é de pacientes adultos/idosos, clínicos, que se encontram em processo pós-operatório de cirurgias de pequena, média e alta complexidade, incluindo cirurgias cardíacas.

A UTI B situa-se em um hospital geral, também privado, de pequeno porte, no município de Vitória da Conquista, e atende toda a microrregião onde está inserida. Ela dispõe de sete leitos destinados ao cuidado de pacientes de alta complexidade terapêutica, sendo sua demanda pacientes adultos/idosos, desde pós-operatórios de cirurgias eletivas e/ou de urgência e emergência, da baixa, média e alta complexidade, até pacientes vítimas de traumas, pós-operatório de cirurgias ortopédicas, cardíacas, neurológicas, pacientes portadores de doenças autoimunes que necessitam de uma terapêutica invasiva e complexa, de tratamento de urgências dialíticas, dentre outros.

Os participantes da pesquisa foram profissionais da equipe de enfermagem que atenderam aos critérios: ser profissional da assistência direta aos pacientes dessas unidades e estar em escala no período da coleta de dados. Não foram aplicados critérios de exclusão.

Para atender aos aspectos éticos, foi respeitada a Resolução no 466/2012 do Conselho Nacional de Saúde ${ }^{(9)}$, e a pesquisa foi aprovada pelo Comitê de Ética da Escola de Enfermagem da Universidade Federal da Bahia, sob parecer 684.286, em 04 de junho de 2014 e CAAE: 2613. 2113.2.0000.5531. Todos que aceitaram participar assinaram o Termo de Consentimento Livre e Esclarecido.

A coleta de dados ocorreu nos meses de julho e agosto de 2014, por meio de entrevista semiestruturada, com duração média de 30 minutos, gravada e validada posteriormente junto aos participantes, cujo instrumento continha 
duas partes: 1) dados de caracterização dos participantes; 2) cinco questões semiestruturadas relativas ao objeto de estudo. Para preservar a identidade dos profissionais, os depoimentos foram numerados, os enfermeiros, identificados pela letra $E$, os técnicos de enfermagem, pela letra $T$, obedecendo à ordem numérica da realização das entrevistas.

As entrevistas foram realizadas pelas pesquisadoras, de forma individual, em uma sala reservada na unidade, após autorização prévia da instituição, do Comitê de Ética em Pesquisa e da concordância dos colaboradores em participar. Todas as entrevistas aconteceram após o turno de trabalho desses profissionais.

A quantidade de participantes não foi determinante na decisão de escolha dos depoimentos e/ou do volume significativo de respostas a serem utilizadas. Essa decisão de escolha dos dados teve relação com o significado das respostas e com a sua importância nas possibilidades de atender aos objetivos do estudo. Para essa definição, as entrevistas foram encerradas após a identificação da saturação do conteúdo temático significativo e emergente nos depoimentos. Os dados obtidos após a transcrição integral realizada pelas autoras foram tratados a partir da Técnica de Análise de Conteúdo Categorial ${ }^{(10)}$, contemplando as etapas de leitura flutuante a partir do corpus dos depoimentos; da seleção das unidades de significado orientada pela questão da pesquisa; e, por fim, do processo de categorização e codificação das unidades de análise. A discussão foi feita a partir de evidências científicas sobre o tema.

\section{RESULTADOS}

Participaram da pesquisa 85 profissionais, sendo 26 enfermeiros e 59 técnicos de enfermagem. Provenientes da UTI A, participaram 21 enfermeiros de um total de 27 e 39 técnicos de enfermagem de um total de 46.Dois enfermeiros recusaram-se a participar, justificando indisponibilidade de tempo para a entrevista após o plantão, e quatro estavam afastados da instituição no período da coleta de dados. Dentre os técnicos de enfermagem, um profissional se recusou a participar da pesquisa e seis estavam afastados da instituição no período da coleta. Já da UTI B, todos os profissionais escalados no período da coleta participaram da pesquisa, sendo cinco enfermeiros e 20 técnicos de enfermagem.

Da leitura e do tratamento dos relatos, emergiram três categorias de análise: Avaliação inicial do paciente com necessidade de restrição, Conhecimento dos profissionais sobre a regulamentação da restrição física e Monitoramento do paciente em uso de restrição física.

\section{Avaliação inicial do paciente com necessidade de restrição}

A avaliação neurológica foi a técnica mais empregada pelos profissionais para verificar a necessidade de restrição física. Um total de 79 dos entrevistados citaram verificar o nível de consciência, o grau de agitação ou o estado de desorientação de seus pacientes durante a avaliação.

Eu observo seu nível de consciência, es-
tado de desorientação e agressividade
para saber se vai precisar conter. (T39)

Avalio o padrão neurológico e a possibilidade de perda de dispositivos. (E16)

Foi evidenciada, também, uma preocupação com a segurança do paciente e com a continuidade da terapêutica sem intercorrências. Nesse sentido, 30 profissionais referiram avaliar se o paciente encontrava-se em risco de 
exteriorização de dispositivos e 11 avaliavam o risco de queda.

Eu avalio a agitação, orientação, se consegue entender a importância dos dispositivos. Se sedado, a gente contém para proteção do paciente. (E15).

Dependendo da lucidez e do estado emocional, ele pode retirar tubos, drenos, etc, trazendo prejuízo para si. (T08).

Tem que ver se o paciente tem risco de queda, exteriorização de dispositivos ou se está em agitação psicomotora. (E14).

As preocupações relativas à segurança do paciente, no que se refere às quedas, à exteriorização de dispositivos e à agressividade, surgem não apenas com relação à segurança do sujeito mas também à segurança da equipe e de outros pacientes. Dentre os entrevistados, 13 profissionais citam agressividade e agitação como justificativas para conter o paciente na UTI.

A gente olha se o paciente está agressivo, em agitação, se ele pode tentar fugir do leito ou retirar acessos. (T09).

Se está agressivo, tem que ver se existe risco dele causar algum dano a si próprio ou à equipe. (E19).

Quando questionados sobre as alternativas empregadas antes de optar pela restrição física, 59 profissionais afirmaram dialogar com o paciente para esclarecer a necessidade de mantê-lo tranquilo e colaborativo com o seu tratamento. $\mathrm{O}$ uso de medicamentos/sedativos para o controle da agitação psicomotora e para a evolução do quadro neurológico foi citado por 12 entrevistados, sendo que seis mencionaram a necessidade de comunicação da equipe multiprofissional para avaliação e estabelecimento de critérios para o emprego do procedimento em questão.

$$
\begin{aligned}
& \text { Observar o motivo pelo qual está } \\
& \text { agitado, conversar, tentar acalmar, } \\
& \text { solicitar ao médico para avaliar, pedir } \\
& \text { orientação à enfermeira para não ser } \\
& \text { utilizada a contenção como primeira } \\
& \text { opção. (T22). }
\end{aligned}
$$

Converso com ele, se ele não obedecer, falo com o médico para fazer medicação, se não obedecer, tem que conter, não pode deixar ele cair da cama. (T30).

Solicito a enfermeira para avaliar o paciente. (T23).

Dentre os entrevistados, cinco afirmaram que optam imediatamente pela restrição física, sem utilizar nenhum método alternativo anteriormente.

Quando é assim, eu prefiro conter logo para evitar problemas para nós depois. (T17).

Tais relatos apontam que os critérios para uso da restrição física devem passar não só pela avaliação inicial de cada paciente, mas também pelo conhecimento sobre o tema e os seus instrumentos legais.

Conhecimento do profissional sobre a regulamentação da restrição física

No que diz respeito ao respaldo legal da categoria de enfermagem, das 85 profissionais 
entrevistadas, 71 desconheciam a resolução do COFEn 427/2012(7) e, embora duas apontassem saber da existência de leis sobre a restrição física, não tinham conhecimento sobre seu conteúdo. Dos profissionais que afirmaram conhecer a resolução, dez eram técnicos de enfermagem e quatro, enfermeiros.

Eu já ouvi falar alguma coisa sobre uma lei aí, mas nunca li. (T7).

Eu sei que tem uma resolução que fala sobre contenção mecânica, que é prescrição médica, mas não sei exatamente o que fala. (E13).

Nunca nem ouvi falar, fala o quê? (T2).

Percebeu-se, também, durante a pesquisa, a ausência de protocolo institucional para o emprego desta terapêutica em ambas as unidades.

Não temos um protocolo para uso das contenções, fazemos de acordo com a rotina da unidade. (E3).

Os relatos apresentados evidenciam a superficialidade ou a ausência de conhecimento da equipe de enfermagem, com relação aos instrumentos legais e aos protocolos concernentes à restrição física.

Monitorização do paciente durante o uso da restrição física

A monitorização do paciente durante o uso da restrição física, de acordo com os discursos, é realizada pela equipe de enfermagem de diversas formas, seja na percepção de alterações físicas e hemodinâmicas durante o uso, seja na monitorização neurológica, atentando quanto à possibilidade de mudança dessa conduta. Esses aspectos foram observados nos depoimentos dos profissionais, quando 29 participantes afirmaram realizar a monitorização por meio de observação contínua. Quando questionados sobre como seria realizada essa vigilância, um profissional comentou:

\begin{abstract}
A presença constante na cabeceira do paciente a cada 30 a 45 minutos, perguntando como ele está no momento.
\end{abstract}

(TE 14).

A avaliação da integridade cutânea foi a segunda forma de monitoramento mais citada pelos entrevistados, e 26 deles referiram essa preocupação. $\mathrm{O}$ aparecimento de lesões de pele foi abordado pelos enfermeiros como um ponto inquietante devido ao conhecimento prévio de possíveis atritos exercidos nos membros durante o emprego da restrição e ao cisalhamento causado, principalmente, em pacientes agitados.

$$
\begin{aligned}
& \text { Cuidado de retirar se a contenção está } \\
& \text { garroteando o membro do paciente. } \\
& \text { Para certificar, coloco meus dedos } \\
& \text { dentro da contenção, se couber minha } \\
& \text { mão entre a contenção e o membro, é } \\
& \text { porque está boa. (E5). }
\end{aligned}
$$

A monitorização neurológica para investigar se ocorreu piora da agitação após uso de restrição física foi outra forma de monitorização citada por 18 profissionais.

A preocupação com alterações hemodinâmicas decorrentes do emprego da restrição física foi evidenciada na fala de sete entrevistados, que ressaltaram a necessidade de garantir o conforto do paciente em uso, citando como critério avaliador o grau de ansiedade do sujeito e a situação circulatória no membro. 
Observo o seu estado de comportamento, se ele está melhorando, se a contenção está apertada, procuro ver a circulação, se tem edema, etc. (T39).

Ademais, dos 85 profissionais entrevistados, quatro técnicos de enfermagem não souberam informar como realizam a monitorização do paciente em uso de restrição física.

Em alguns casos, é difícil monitorar. (T38).

Não sei informar. (T4).

Observo pelo monitor. (T10 e T15).

Percebe-se, assim, que a monitorização é feita de forma individual, a partir da experiência e do conhecimento de cada profissional, e que menos da metade dos participantes seguem os critérios de monitorização estabelecidos pelo COFEn.

\section{DISCUSSÃO}

Alguns dos resultados encontrados e reforçados por outros estudos apontam que, em geral, os motivos para uso da restrição física estão atrelados às ações de proteção para o paciente e para a equipe, bem como para garantir continuidade do tratamento, evitando-se, principalmente, a perda de dispositivos $\mathrm{s}^{(11,12,13)}$.

Um estudo realizado em Hong Kong mostrou que o principal critério para o uso de restrição física em pacientes hospitalizados foi a garantia da sua segurança, colocada como fator principal para implementação da restrição. Nesse sentido, uma das justificativas apontadas foi a escassez de enfermeiras para garantirem um ambiente seguro, fazendo com que os profissio- nais utilizassem a restrição física como primeira alternativa terapêutica ${ }^{(14)}$.

A segurança do paciente é de responsabilidade de toda a equipe, mas o corpo de enfermagem, por estar mais tempo em contato com o sujeito, acaba sendo responsabilizado por danos sofridos durante o tempo de hospitalização. Isso faz com que os profissionais optem, de imediato, por conter o paciente, considerando a restrição física como primeira medida de proteção, o que a coloca como uma solução, e não um problema.

Algumas terapêuticas, muitas vezes usadas para garantir uma "segurança" ao paciente e não o expor a riscos de perda de dispositivos e de quedas, são controversas, uma vez que não há evidências na literatura apontando a restrição física como proteção contra quedas ou como benéfica ao paciente.

Um estudo internacional abrangente, de revisão sistemática sobre o tema, revelou que seu uso em pacientes hospitalizados pode estar associado ao aumento do tempo de hospitalização; à ocorrência de infecções, principalmente em restrições prolongadas por mais que quatro dias; a alterações decorrentes de imobilidade, quedas, morte $^{(15)}$, declínio cognitivo, desorientação, dupla incontinência, aumento da incidência de úlceras por pressão, da agitação e da dependência para cuidar das necessidades pessoais diárias ${ }^{(14)}$, além de ser causa frequente de estresse pós-traumático associado ao internamento na UTI, sobretudo em pacientes agitados, estressados, ansiosos, temerosos e com dificuldades de se expressar ${ }^{(16)}$. Em situações de risco de queda, embora a utilização da restrição física pareça ser uma boa estratégia preventiva, ela deve ser utilizada como estratégia de prevenção somente como último recurso. Outras ações importantes incluem mobilização precoce e esforços para preservar a força muscular do paciente ${ }^{(13)}$ e a adaptação dele ao ambiente , estimulando a autonomia e prevenindo confusão mental e agitação. 
Ademais, o uso da restrição pode acarretar confusão mental como o delírium, especialmente em pacientes com idade avançada ou com disfunções mentais, podendo também ser aplicada nessas situações, principalmente quando hiperativo. Um estudo mostrou que essa disfunção está fortemente associada ao uso da restrição física e à sua duração ${ }^{(17)}$. Outra pesquisa que objetivou analisar o conhecimento de enfermeiros de UTI sobre o delírium apresenta, por meio do Discurso do Sujeito Coletivo, falas relacionadas ao uso das restrições físicas nesses pacientes como medida de segurança, contudo sem critérios de avaliação e/ou monitorização e sem o conhecimento devido dos profissionais de enfermagem em relação às suas consequências ${ }^{(18)}$. Assim, a detecção precoce do delírium e sua prevenção seriam fatores importantes para evitar a restrição.

No ambiente de terapia intensiva, a agressividade e a agitação podem ocorrer com muitos pacientes, por estarem confusos, desorientados, em situação de hipóxia, com afecções neurológicas, entre outros fatores. Nesses casos necessita-se da avaliação da causa desse comportamento antes de realizar a contenção, seja ela química, seja ela mecânica. Essa situação poderia ser melhor conduzida por meio de protocolos institucionais e de treinamentos de equipe.

A avaliação e o manejo de um paciente potencialmente agressivo ou violento são tarefas complexas que exigem dos profissionais habilidades diversas. A prática de restrição física nesses pacientes é controversa, haja vista seu uso maciço e desenfreado em caráter coercitivo/punitivo(5). Sobre isso, estudo realizado no Egito $^{(2)}$ demonstrou que havia uma dificuldade de comunicação entre equipe, pacientes e familiares sobre o critério motivacional para o uso de restrição física, salientando que, quando há desconhecimento dos motivos que justifiquem tal terapêutica, ocorre aumento da ansiedade e do comportamento agressivo.
Observa-se, assim, a importância da comunicação entre paciente, profissional e família, a fim de esclarecer toda a situação que o paciente está vivenciando e os motivos que envolvem o emprego da restrição física. Caso o paciente não colabore, os familiares, por sua vez, poderão ser informados da necessidade do procedimento e solicitados para contribuir na minimização da ansiedade e da agressividade que possam ocorrer pelo emprego da restrição.

A restrição física deve ser o último recurso utilizado, ou seja, apenas nos casos em que alternativas menos restritivas tentadas anteriormente não obtiverem sucesso. Ademais, seu uso deve ser justificado por critérios fundamentados e estruturados ${ }^{(19)}$. Então, sendo necessária a restrição física, ela deve ser feita por equipe treinada para sua monitorização efetiva.

Após a sua aplicação, a monitorização constante é uma ação imprescindível devido à grande ocorrência de eventos adversos. Diante disso, justifica-se a preocupação dos entrevistados com a vigilância relacionada à integridade cutânea desses pacientes, visto que as restrições físicas são responsáveis pela ocorrência de diversas lesões de pele, como rubor, escoriações e edema local. Para diminuir esse risco, muitas unidades, inclusive as unidades lócus, têm se utilizado de contenções feitas com tecido menos agressivo à pele, substituindo as ataduras. Essa é uma preocupação importante, já que muitos desses pacientes contidos são idosos, que estão debilitados, e, por isso, com maior propensão de danos relacionados à pele.

A Resolução do COFEn ${ }^{(7)}$ aponta a necessidade de avaliar a contenção a cada hora, observando se ainda existe motivo para mantê-la e monitorando o paciente em relação a seu estado de orientação, hemodinâmica, condições da pele, perfusão periférica, alterações da mobilidade, higiene entre outras possíveis alterações. Essa monitorização deve ser mais acentuada em 
se tratando de pacientes sedados ou sonolentos, idosos, crianças, adolescentes ou pacientes com problemas clínicos.

Por outro lado, o estudo em questão aponta a falta de conhecimento dos profissionais a respeito das bases legais para esse procedimento. Assim, mesmo que estas justifiquem o uso das restrições em decisões paralelas, a falta de conhecimento pode expor a equipe ao risco de imperícia, impudência e negligência, podendo causar lesões no paciente e possíveis penalidades para os profissionais de enfermagem envolvidos, que vão desde advertência verbal até cassação(7,11).

Além disso, a mesma Resolução aponta que, ao se decidir pela implantação da terapêutica restritiva, exceto em situações de urgência/ emergência, deve haver uma supervisão direta do enfermeiro. A restrição deve ser prescrita pelo médico e guiada por protocolos estabelecidos pelas instituições, o que não foi observado nas unidades lócus ${ }^{(20)}$. Orienta-se que a contenção só seja aplicada com avaliação rigorosa e global do paciente, baseada no julgamento clínico, e que jamais ocorra como punição ou intimidação(20). Logo, essa avaliação e esse monitoramento dos casos tendem a diminuir a necessidade de contenção e o tempo de seu uso, reduzindo, consequentemente, as iatrogenias naqueles que estão contidos.

Essa informação reforça a necessidade de esclarecimento e de treinamento da equipe de enfermagem, a fim de que se possa modificar a cultura da contenção mecânica para uma de não contenção, procurando rever indicações e buscando melhorar o ambiente e o cuidado na terapia intensiva, de forma que o tratamento se torne mais acolhedor, para oferecer mais tranquilidade ao paciente. Estudos mostram que a educação permanente da equipe pode reduzir o uso das restrições ${ }^{(14,17)}$. Sobre isso, uma pesquisa de intervenção com um programa de redução das restrições físicas, por meio da educação dos profissionais e da criação de um comitê para redução da restrição, mostrou a importância dessas ferramentas no controle desse procedimento ${ }^{(14)}$.

Além disso, são importantes a criação de protocolos institucionais e o aprimoramento das equipes multidisciplinares para melhor manejo dos pacientes na terapia intensiva, bem como estímulos para conhecimentos e esclarecimentos das bases legais que envolvem essa temática.

O estudo, naturalmente, apresenta limitações: indisponibilidade de alguns profissionais das unidades em participar, reduzindo a amostra avaliada, teor das conclusões e pressupostos pautados em discursos humanos passíveis de reformulações prévias antes de sua exposição ao interlocutor, e sem possibilidade de avaliação do nível de consonância com a prática dos mesmos, já que não foi realizada observação em campo. Entretanto, tais limitações não influenciaram na qualidade dos dados obtidos, na coerência dos achados com as produções publicadas sobre o assunto e, tampouco, na percepção da forma como os profissionais de enfermagem manejam as restrições nos pacientes críticos.

Ademais, o estudo aponta para a forma negligenciada como as restrições físicas vêm sendo tratadas dentro das UTIs e para o importante papel que o enfermeiro intensivista tem no acompanhamento desses pacientes e na decisão sobre a necessidade ou não do procedimento, carecendo, porém, de informação e treinamento.

\section{CONCLUSÃO}

O estudo revelou que as restrições físicas em UTI têm sido realizadas sem homogeneidade no que tange aos critérios para instalação e monitoramento durante o uso; tal realidade pode estar relacionada à ausência de protocolos 
institucionais, de treinamentos de equipe e do conhecimento dos profissionais de instrumentos legais relacionados à temática.

Sobre os critérios para uso, o receio da perda de dispositivos, acompanhado pelo risco de queda, foram fatores preponderantes nos discursos para sua aplicação, após insucesso de outras intervenções, seguido do fator "agitação psicomotora".

A respeito da monitorização dos pacientes contidos, os profissionais referiram que a "avaliação neurológica" é a técnica mais utilizada para percepção de alterações durante o uso e de necessidade de mantimento ou suspensão da restrição. Além disso, declaram também observar o estado da pele e a hemodinâmica. Entretanto, em ambas as categorias profissionais, não havia bases definidas para a avaliação de cada profissional, com atitudes isoladas e baseadas em concepções pessoais e empíricas.

Recomenda-se, portanto, a criação de protocolos institucionais padronizados e de treinamentos da equipe multiprofissional para, assim, proporcionar abordagens dessa técnica com segurança e qualidade, além da constante vigilância do ambiente e dos recursos humanos na terapia intensiva, a fim de torná-lo acolhedor e humanizado.

Por fim, acredita-se que esta pesquisa possa contribuir com reflexões, por parte das equipes de saúde, sobre a necessidade de o paciente ser restrito ao leito, bem como suas consequências.

\section{REFERÊNCIAS}

1. Ministério da Saúde (BR). Portaria no 2.338 de 3 de outubro de 2011, Estabelece diretrizes e cria mecanismos para a implantação do componente Sala de Estabilização (SE) da Rede de Atenção às Urgências. [Internet]. 2011 [cited 2015 Apr 9]. Available from: http://bvsms.saude.gov.br/bvs/ saudelegis/gm/2011/prt2338_03_10_2011.html

2. Kandeel NA, Attia ALK. Physical restraints practice in adult intensive care units in Egypt. Nurs Heal Sci. 2013 Mar;15(1):79-85.

3. Amatriain AIPC, Olmedo AN, Viguria RG, Martinez ER, Coscojuela MAM, Assian Erro MC. Restricciones físicas en UCl: su utilización y percepción de pacientes y familiares. Enferm Intensiva. 2012 Apr ;23(2):77-86.

4. Coutinho MHB, Santos SRG. Manual de procedimentos de enfermagem. 1st ed. Brasília: Secretaria de Saúde; 2012.

5. Mantovani C, Migon MN, Alheira FV, Del- Ben CM. Managing agitated or aggressive patients. Rev Bras Psiquiatr. 2010 Out ;32(Supl II):96-103.

6. Mussel E, Ribeiro VF, Leite LF, org. Contenção Física dos Pacientes em Quadro de Agitação Psicomotora. Diretrizes Clínicas - Procedimento Operacional Padrão - Protocolo Clínico. Fundação Hospitalar do Estado de Minas Gerais. 2012 Jan.

7. Conselho Federal de Enfermagem (COFEn). Normatização dos procedimentos da enfermagem no emprego de contenção mecânica de pacientes. Resolução no 427/2012 [Internet]. 2012 [cited 2015 Apr 14]. Available from: http://www.cofen. gov.br/resoluo-cofen-n-4272012_9146.html

8. Ministério da Saúde (BR). Portaria no. 529, de 1 de abril de 2013. Institui o Programa Nacional de Segurança do Paciente (PNSP) [Internet]. Diário Oficial da União 1 abr 2013. [cited 2014 Apr 2]. Available from: http://bvsms.saude.gov.br/bvs/ saudelegis/gm/2013/prt0529_01_04_2013.html

9. Ministério da Saúde (BR). Resolução número 466 de 12 de dezembro de 2012, trata de pesquisa e testes com seres humanos [Internet]. Diário Oficial da União 12 de dezembro de 2012. [cited 2015 Apr 14]. Available from: http://conselho. saude.gov.br/resolucoes/2012/Reso466.pdf

10. Bardin L. Análise de conteúdo. São Paulo: Edições 70; 2011.

11. Conselho Federal de Enfermagem (COFEN). Parecer técnico - Contenção mecânica no032/2009 [Internet]. 2009 [cited 2015 Apr 14]. Available from: http://www.coren-df.gov.br/portal/index. php/pareceres/80-pareceres-cofen/688-parecer-de-relatora-no-0322009

12. Fillipi J, Flores A, Bettinelli LA, Pomatti DM. A equipe multiprofissional frente ao uso da contenção 
mecânica. Rev. Contexto \& Saúde. 2011 Jan/Jun ;20(10):573-8.

13. Watcher R. Compreendendo a segurança do paciente. São Paulo: Artmed; 2013.

14. Lai CKY, Chow SKY, Suen LKP, Wong IWC. The Effect of a Restraint Reduction Program on Physical Restraint Rates in Rehabilitation Settings in Hong Kong. Rehabil Res Pr. 2011, ID 284604; 2011:9.

15. Evans D, Wood J, Lambert L. Patient injury and physical restraint devices: a systematic review .J Adv Nurs. 2003;41(3): 274-82.

16. Heather WH, Howland L. Posttraumatic Stress Syndrome Associated With Stays in the Intensive Care Unit: Importance of Nurses' Involvement. Crit Care Nurse. 2015 jun ;35(3): 44-54.

17. Andrews L, Silva SG, Kaplan S, Zimbro K. Delirium monitoring and patient outcomes in a general intensive care unit. Am J Crit Care. 2015;24(1):48-56.

18. Ribeiro $\mathrm{SCL}$, Nascimento ERP, Lazzari DD, Jung W, Boes AA, Bertoncello KC. Knowledge of nurses about delirium in critical patients: collective subject discourse. Text Context Nurs. 2015;24(2):513-20.

19. Filippi J, Flores A, Bettinelli LA, Pomatti DM. A equipe multiprofissional frente ao uso da contenção mecânica. Rev Context e Saúde. 2011;10(20):573-8.

20. Braga IP, Souza de JC, Leite MB, Fonseca B, Silva EM, Volpe FM. Physical restraint in a Brazilian psychiatric hospital: cross-sectional study of practice and risk factors. J bras Psiquiatr. 2016;65(1):53-9.
Todos os autores participaram das fases dessa publicação em uma ou mais etapas a seguir, de acordo com as recomendações do International Committe of Medical Journal Editors (ICMJE, 2013): (a) participação substancial na concepção ou confecção do manuscrito ou da coleta, análise ou interpretação dos dados; (b) elaboração do trabalho ou realização de revisão crítica do conteúdo intelectual; (c) aprovação da versão submetida. Todos os autores declaram para os devidos fins que são de suas responsabilidades o conteúdo relacionado a todos os aspectos do manuscrito submetido ao OBJN. Garantem que as questões relacionadas com a exatidão ou integridade de qualquer parte do artigo foram devidamente investigadas e resolvidas. Eximindo, portanto o OBJN de qualquer participação solidária em eventuais imbróglios sobre a matéria em apreço. Todos os autores declaram que não possuem conflito de interesses, seja de ordem financeira ou de relacionamento, que influencie a redação e/ou interpretação dos achados. Essa declaração foi assinada digitalmente por todos os autores conforme recomendação do ICMJE, cujo modelo está disponível em http://www. objnursing.uff.br/normas/DUDE_final_13-06-2013.pdf

Recebido: 01/05/2016

Revisado: 22/02/2017

Aprovado: 07/03/2017 